\title{
\begin{tabular}{l|l} 
pcori)? & PATIENT-CENTERED OUTCOMES RESEARCH INSTITUTE \\
RESEARCH SUMMARY
\end{tabular}
}

\section{Adding a New Role at Clinics to Help Patients Access Community Resources}

Principal investigator

Clarissa Hsu, PhD
Organization

Kaiser Foundation Health Plan of Washington

\section{What was the research about?}

Chronic, or long-term, health problems such as diabetes or high blood pressure are on the rise in the United States. Doctors can help patients manage chronic health problems like these. Community programs may also help people manage their health. For example, community programs may give people free rides to the doctor or teach people how to make healthy meals.

In this study, the research team created a new job within the health care teams at primary care clinics called a community resource specialist, or CRS. CRSs were non-medical professionals who had expertise in community resources. The CRS helped patients find programs and resources in their communities that supported their health goals. The CRS also worked with patients to make a plan for using those resources. After three months, the team wanted to see how the CRS affected both patients and clinic staff.

\section{What were the results?}

- On surveys, patients reported that they were very satisfied with the services they received from the CRS.

- In interviews, patients who saw the CRS reported improved health and health habits. But the research team didn't find any changes in these patients' health or health habits on survey responses.

- Patients who saw the CRS used some types of healthcare services, such as going to the doctor or hospital, slightly more than patients who didn't see the CRS.

- In interviews, clinic staff who worked with a CRS reported that their workload got easier and they could provide better care to patients with a CRS on the team.

\section{Who was in the study?}

Patients in the study were from three Kaiser Permanente clinics in Washington State. Of the 622 patients referred to the CRS, 418 patients had at least one complete visit with the CRS. Common health problems for these patients were diabetes, high blood pressure, or depression. Of these 418 patients, 73 percent were over the age of 35 , and 75 percent were female. Patients in the study were racially diverse.

\section{What did the research team do?}

Before the study, the research team met with 12 patients, 11 providers, and clinic staff at a four-day workshop to create the CRS job. Then, three clinics in a large healthcare system tested the role. Doctors in these clinics referred patients to the CRS.

The research team gave a survey to patients during their referral and again three months later. Next, the team had a group discussion with patients and talked with clinic staff about the CRS role. Finally, using electronic health records, the research team looked at patients' use of healthcare services three and six months after they saw a CRS. The team compared these health records to health records of similar patients that did not see a CRS. 


\section{What were the limits of the study?}

There were several problems that affected the study, including staff turnover. These problems required the research team to change the study design and made it difficult to evaluate the CRS role as planned.

\section{How can people use the results?}

Clinics serving patients with chronic illnesses may look at whether patients have adequate access to community resources and figure out how best to support them.

To learn more about this project, visit pcori.org/Hsu089. 